\title{
NORMALITY AND ABNORMALITY IN THE CALCULUS OF VARIATIONS*
}

BY

\author{
G. A. BLISS
}

Within the past few years a number of papers concerning the problem of Bolza in the calculus of variations have been published which make it possible to carry through the theory of this problem with much simplified assumptions concerning what is called the normality of the minimizing arc. I refer especially to papers by Graves [8], $\dagger$ Hestenes [11, 14, 16], Reid [15], and Morse [13]. These papers and others are also important because they bring the theory of problems of the calculus of variations with variable end points to a stage comparable with that already attained for the more special case in which the end points are fixed.

In the theories of Bolza [1, chap. 11, 12] and Bliss [2] for the problem of Lagrange with fixed end points it was assumed that the minimizing arc considered, extended slightly at both ends, was normal on every sub-interval. Morse [4] showed that the theory could be carried through on the assumption that the arc itself, without extensions, was normal on every sub-interval. The most important case, however, turns out to be the one for which the arc as a whole is normal relative to the problem considered, but not necessarily normal on sub-intervals. Graves proved the necessary condition of Weierstrass for such a normal minimizing arc, and Hestenes deduced further necessary conditions and gave sufficiency proofs for a minimum. The importance of these results is emphasized by the fact that for the very general problem of Mayer, which may be regarded as a sub-case of the problem of Bolza, every minimizing arc is abnormal on every sub-interval, even though the arc as a whole is normal relative to the problem. Thus the problem of Mayer needs a separate treatment, such as was given by Bliss and Hestenes [9, 10], unless one has at his command results equivalent to the recent extensions of the theory of the problem of Bolza mentioned above.

In this paper I am attempting to analyze, more explicitly than has been done before, the meaning of normality and abnormality for the calculus of variations. To do this I have emphasized in $\$ 1$ below the meaning of normality for the problem of a relative minimum of a function of a finite number of variables. In $\$ 2$ analogous notions are discussed for problems of the cal-

* Presented to the Society, April 20, 1935; received by the editors April 1, 1937.

† The numbers in brackets here and elsewhere refer to the bibliography at the end of this paper. 
culus of variations. From this discussion it will be clear that a normal arc for the problem of Bolza is a non-singular arc of the class in which a minimizing one is sought. The singular arcs of the class are the abnormal ones. They have an enormous variety of types. It is not likely that a general theory can be formulated which would apply to all of them, though one might characterize and study successfully some very general cases.

In the papers of Graves and Hestenes mentioned above there is no explicit assumption concerning normality. The arc studied is assumed only to have a set of multipliers like those which it would have if it were normal for the problem of Bolza considered. In the following pages it will be seen that, though such an arc may be abnormal for the problem originally considered, it is nevertheless normal for a second problem of Bolza obtained from the first by suitably extending the class of arcs in which a minimizing one is sought. Furthermore the properties characterizing a minimizing arc for the original problem are effective for the second, so that the sufficiency theorems of Hestenes for arcs which are normal have as easy consequences those for the abnormal arcs permitted by his hypotheses. This makes possible a number of simplifications in the details of the proofs. It is not to be expected, of course, that new necessary conditions on a minimizing arc can be secured by extending the class of arcs in which a minimizing one is sought. The paper of Graves, therefore, seems to contain results not attainable by considering only normal arcs.

In the introduction to his paper [13] Morse makes a statement concerning priority for the proofs of sufficiency theorems without assumptions of normality which might easily be misunderstood and about which I should like to make the following comments. Hestenes had previously proved, in his paper [11], three sufficiency theorems (Theorems 9:1, 9:3, 9:5) without explicit assumptions of normality, and also a fourth theorem (Theorem 9:4) with normality assumptions still undesirably strong, but weaker than those which had before been used. Reid [15] and Morse [13] showed independently that by means of a further lemma, but aided still essentially by the results of Hestenes, this fourth theorem can be brought to a par with the others. The condition $\mathrm{VI}^{\prime}$ [11, p. 811] of Theorem 9:4 is analogous to one which I used in the paper [5], and which was originally due to A. Mayer. Its statement involves the notion of conjugate points and is therefore more closely related to the classical conditions of Jacobi for simpler problems than the corresponding conditions of the other theorems. I think it should be understood that the priority comment of Morse is applicable to Theorem 9:4 of Hestenes, but not to the other three theorems of his paper, which are equally important. I may add that the theorems of Hestenes were proved with great originality 
and ingenuity while he was my research assistant at the University of Chicago in 1933 [16, p. 543]. When he went away he left a manuscript with me in which the theorems were, at my suggestion, deduced only for normal arcs, the ones which then, as well as now, seemed to me the most important, even though the justification of the arguments of the present paper was at that time missing. This manuscript has since appeared in much modified form in my mimeographed lectures on the problem of Bolza [12]. In his paper [11] Hestenes showed that his methods are also effective for the problem of Bolza in the form adopted by Morse.

1. Abnormality for minima of functions of a finite number of variables. The significance of the notion of abnormality in the calculus of variations can be indicated by a study of the theory of the simpler problem of finding, in the set of points $y=\left(y_{1}, \cdots, y_{n}\right)$ satisfying a system of equations of the form

$$
\phi_{\beta}(y)=0(\beta=1, \cdots: m<n),
$$

one which minimizes a function $f(y)$. For a point $y^{0}=\left(y_{1}^{0}, \cdots, y_{n}^{0}\right)$ near which the functions $f$ and $\phi_{\beta}$ have continuous partial derivatives of at least the second order, and which satisfies the equations $\phi_{\beta}=0$, we have the following theorems, some of which are, of course, well known.

TheOREM 1:1. A first necessary condition for $f\left(y^{0}\right)$ to be a minimum is that there exist constants $l_{0}, l_{\beta}$ not all zero such that the derivatives $F_{v_{i}}$ of the function

all vanish at $y^{0}$.

$$
F=l_{0} f+l_{\beta} \phi_{\beta}
$$

To prove this we have only to note that the determinants of the matrix

$$
\left\|\begin{array}{c}
f_{y_{i}}\left(y^{0}\right) \\
\phi_{\beta y_{i}}\left(y^{0}\right)
\end{array}\right\|
$$

must all vanish. Otherwise, according to well known implicit function theorems, the equations $f(y)=f\left(y^{0}\right)+u, \phi_{\beta}(y)=0$ would have solutions $y$ for negative values of $u$, and $f\left(y^{0}\right)$ could not be a minimum.

A point $y^{0}$ has by definition order of abnormality equal to $q$ if there exist $q$ linearly independent sets of multipliers of the form $l_{0}=0, l_{\beta}$ having the property of the theorem. When $q=0$ the point $y^{0}$ is said to be normal. A necessary and sufficient condition for abnormality of order $q$ is evidently that the matrix $\left\|\phi_{\beta y_{i}}\left(y^{0}\right)\right\|$ have rank $m-q$. At a normal point $y^{0}$ the multipliers $l_{0}, l_{\beta}$ of the theorem can be divided by $l_{0}$ and put into the form $l_{0}=1, l_{\beta}$. In this form they are unique, since the non-vanishing difference of two such sets would be a set of multipliers implying abnormality. 
LEMMA 1:1. If a point $y^{0}$ is normal, then for every set of constants $\eta_{i}$ $(i=1, \cdots, n)$ satisfying the equations

$$
\phi_{\beta y_{i}}\left(y^{0}\right) \eta_{i}=0
$$

there exists a set of functions $y_{i}(b)$ having continuous second derivatives near $b=0$, satisfying the equations $\phi_{\beta}=0$, and such that

$$
y_{i}(0)=y_{i}{ }^{0}, y_{i}^{\prime}(0)=\eta_{i} \text {. }
$$

The proof can be made by considering the equations

$$
\phi_{\beta}(y)=0, \phi_{\gamma}(y)=\phi_{\gamma}\left(y^{0}\right)+b \zeta_{\gamma} \quad(\beta=1, \cdots, m ; \gamma=m+1, \cdots, n)
$$

in which the auxiliary functions $\phi_{\gamma}(y)$ are selected so that they have continuous second derivatives near $y^{0}$ and make the functional determinant $\left|\phi_{i y k}\left(y^{0}\right)\right|$ different from zero, and in which the constants $\zeta_{\gamma}$ are defined by the equations

$$
\phi_{\gamma y_{i}}\left(y^{0}\right) \eta_{i}=\zeta_{\gamma} .
$$

Equations $(1: 2)$ then have solutions $y_{i}(b)$ with continuous derivatives of at least the second order near $b=0$, and such that $y_{i}(0)=y_{i}{ }^{0}$. By differentiating with respect to $b$ the equations $(1: 2)$ with these solutions substituted, we find the equations

$$
\begin{aligned}
& \phi_{\beta y_{i}}\left(y^{0}\right) y_{i}^{\prime}(0)=0, \\
& \phi_{\gamma y_{i}}\left(y^{0}\right) y_{i}^{\prime}(0)=\zeta_{\gamma} .
\end{aligned}
$$

With equations $(1: 1)$ and $(1: 3)$ these show that $y_{i}^{\prime}(0)=\eta_{i}$.

THEOREM 1:2. If $y^{0}$ is a normal point and $f\left(y^{0}\right)$ a minimum then the condition

$$
F_{y_{i} y_{k}}\left(y^{0}\right) \eta_{i} \eta_{k} \geqq 0
$$

must hold for every set $\eta_{i}$ satisfying the equations (1:1), where $F=f+l_{\beta} \phi_{\beta}$ is the function formed with the unique set of multipliers $l_{0}=1, l_{\beta}$ belonging to $y^{0}$.

The conclusion of the theorem is due to the fact that the function $g(b)=f[y(b)]$, formed with the functions $y_{i}(b)$ of the lemma, must have a minimum at $b=0$. Since

$$
\phi_{\beta y_{i}}[y(b)] y_{i}^{\prime}(b)=0
$$

the derivatives of $g(b)$ are seen to have the values 


$$
\begin{aligned}
g^{\prime}(b) & =f_{y_{i}}[y(b)] y_{i}^{\prime}(b)=F_{y_{i}}[y(b)] y^{\prime}(b), \\
g^{\prime \prime}(0) & =F_{y_{i} y_{k}}\left(y^{0}\right) \eta_{i} \eta_{k},
\end{aligned}
$$

and for $g(0)$ to be a minimum we must have $g^{\prime \prime}(0) \geqq 0$.

THEOREM 1:3. If a point $y^{0}$ has a set of multipliers $l_{0}=1, l_{\beta}$ for which the function $F=f+l_{\beta} \phi_{\beta}$ satisfies the conditions

$$
F_{y_{i}}\left(y^{0}\right)=0, \quad F_{y_{i} y_{k}}\left(y^{0}\right) \eta_{i} \eta_{k}>0
$$

for all sets $\eta_{i}$ satisfying the equations

$$
\phi_{\beta \nu_{i}}\left(y^{0}\right) \eta_{i}=0,
$$

then $f\left(y^{0}\right)$ is a minimum.

This can be proved with the help of Taylor's formula with integral form of remainder. For every point $y$ near $y^{0}$ satisfying the equations $\phi_{\beta}=0$ we have the equations

$$
\begin{aligned}
f(y)-f\left(y^{0}\right) & =f_{y_{i}}\left(y^{0}\right) \eta_{i}+\int_{0}^{1}(1-\theta) f_{y_{i} y_{k}}\left(y^{\prime}\right) \eta_{i} \eta_{k} d \theta, \\
0 & =\phi_{\beta y_{i}}\left(y^{0}\right) \eta_{i}+\int_{0}^{1}(1-\theta) \phi_{\beta y_{i} y_{k}}\left(y^{\prime}\right) \eta_{i} \eta_{k} d \theta, \\
0 & =\int_{0}^{1} \phi_{\beta y_{i}}\left(y_{i}^{\prime}\right) \eta_{i} d \theta,
\end{aligned}
$$

where $y_{i}^{\prime}=y_{i}^{0}+\theta\left(y_{i}-y_{i}^{0}\right), \eta_{i}=y_{i}-y_{i}{ }^{0}$. From these we find readily

$$
f(y)-f\left(y^{0}\right)=\int_{0}^{1}(1-\theta) F_{y_{i} \nu_{k}}\left(y^{\prime}\right) \eta_{i} \eta_{k} d \theta .
$$

Since the quadratic form in the integrand of the last integral, thought of as a function of independent variables $y^{\prime}$ and $\eta$, is positive for $y^{\prime}=y^{0}$ and all sets $\eta$ satisfying the equations $(1: 5)$, it will remain positive for $y^{\prime}=y^{0}+\theta\left(y-y^{0}\right)$ and all sets $\eta$, including $\eta=y-y^{0}$, satisfying equations (1:6), provided that $y$ lies in a sufficiently small neighborhood $N$ of the point $y^{0}$. Thus we see that for all points $y$ in $N$ satisfying the equations $\phi_{\beta}=0$ the difference $f(y)-f\left(y^{0}\right)$ is positive.

The last theorem is analogous to the sufficiency theorems of Hestenes in the calculus of variations. In it there is no explicit assumption concerning the normality or abnormality of the point $y^{0}$. If $y^{0}$ has abnormality of order $q$, however, let $\nu$ be a variable which ranges over a subset of $m-q$ of the numbers $1, \cdots, m$ such that the matrix $\left\|\phi_{\nu y_{i}}\left(y^{0}\right)\right\|$ has rank $m-q$, and let $\rho$ range over the complementary subset. Then we have the following theorem: 
ThEOREM 1:4. Let $y^{0}$ be a point which satisfies the hypotheses of Theorem $1: 3$ with a set of multipliers $l_{0}=1, l_{\beta}$, and let $\nu$ and $\rho$ be variables having the ranges described in the last paragraph. Then $y^{0}$ is normal for the modified problem of minimizing the function $g=f+l_{\rho} \phi_{\rho}$ in the class of points $y$ satisfying the restricted system of equations $\phi_{\nu}=0$, and $y^{0}$ satisfies the hypotheses of Theorem $1: 3$ for the modified problem with the multipliers $l_{0}=1, l_{v}$. Furthermore if $g\left(y^{0}\right)$ is a minimum for the modified problem, then $f\left(y^{0}\right)$ is a minimum for the original one.

We see that the point $y^{0}$ is normal for the modified problem, since the matrix $\left\|\phi_{\nu y_{i}}\left(y^{0}\right)\right\|$ has rank $m-q$. For the function $F=g+l_{\nu} \phi_{\nu}=f+l_{\beta} \phi_{\beta}$ of the modified problem the conditions (1:4) are satisfied for all sets $\eta$ satisfying the equations

$$
\phi_{\nu y_{i}}\left(y^{0}\right) \eta_{i}=0
$$

since equations $(1: 5)$ are linear and have a matrix of coefficients of rank $m-q$ and hence are consequences of equations (1:7). The set of points $y$ satisfying the equations $\phi_{\nu}=0$ includes the points satisfying the complete system $\phi_{\beta}=0$ as a subclass in which $g=f$. Hence if $g\left(y^{0}\right)$ is a minimum for the modified problem, the value $f\left(y^{0}\right)=g\left(y^{0}\right)$ must have the same property for the original problem.

From the last theorem it is evident that generality is not lost by proving Theorem 1:3 only for points $y^{0}$ which are normal. Such points are, in fact, the non-singular points of the class which satisfy the equations $\phi_{\beta}=0$. Near each of them there are infinitely many points of the class, as is shown by Lemma 1.1, and the minimum problem near one of them is therefore never trivial. Abnormal points, on the other hand, are the singular points of the class, and may occur in a wide variety of types. For some of these points the minimum problem is trivial, as, for example, in the case of a point $y^{0}$, for which $\phi_{1}=0$, which minimizes the function $\phi_{1}$ in the class of points $y$ satisfying the equations $\phi_{2}=\cdots=\phi_{m}=0$. Near such a point $y^{0}$ there is no other point satisfying all of the equations $\phi_{\beta}=0$.

An idea of the great variety of types of abnormal points may be gained by considering the problem of minimizing a function $f\left(y_{1}, y_{2}\right)$ of two variables in the class of points $\left(y_{1}, y_{2}\right)$ satisfying a single equation $\phi\left(y_{1}, y_{2}\right)=0$. The variety of abnormal points possible in this case is at least as great as the variety of singular points of an algebraic curve. The particular example $f=2 y_{1}^{2}-y_{2}^{2}, \phi=y_{1}^{2} y_{2}-y_{2}^{3}=0$, with minimizing point $(0,0)$, shows that the condition involving the quadratic form in Theorem $1: 3$ is not in general necessary for a minimum. 
2. Abnormality in the calculus of variations. The problem to be considered in this section $[12$, p. 4$]$ is that of finding in a class of arcs

$$
y_{i}(x)
$$

$$
\left(i=1, \cdots, n ; x_{1} \leqq x \leqq x_{2}\right)
$$

satisfying conditions of the form

$$
\begin{aligned}
\phi_{\beta}\left(x, y, y^{\prime}\right) & =0 & & (\beta=1, \cdots, m<n), \\
\psi_{\mu}\left[x_{1}, y\left(x_{1}\right), x_{2}, y\left(x_{2}\right)\right] & =0 & & (\mu=1, \cdots, p \leqq 2 n+2)
\end{aligned}
$$

one which minimizes a sum

$$
J=g\left[x_{1}, y\left(x_{1}\right), x_{2}, y\left(x_{2}\right)\right]+\int_{x_{1}}^{x_{2}} f\left(x, y, y^{\prime}\right) d x .
$$

A set of values $\left(x, y, y^{\prime}\right)$ and end values $\left[x_{k}, y_{i s}\right]=\left[x_{s}, y_{i}\left(x_{s}\right)\right](s=1,2)$ is said to be admissible if it lies interior to a region of such values in which the functions $f, g, \phi_{\beta}, \psi_{\mu}$ have continuous derivatives of at least the fourth order, and in which the matrix $\left\|\phi_{\beta \boldsymbol{y}_{i}}\right\|$ and the matrix of first derivatives of the functions $\psi_{\mu}$ have ranks $m$ and $p$, respectively. An admissible arc $C$ defined by functions of the form (2:1) is one which is continuous and consists of a finite number of sub-arcs with continuously turning tangents, and whose elements $\left(x, y, y^{\prime}\right)$ and end values are admissible. When convenient we may represent by $J(C)$, $g(C), \psi_{\mu}(C)$ the values of these functions determined by the arc $C$.

The conditions involved in the sufficiency theorems for this problem are the following, the numbering being that which $I$ have often used [see, e.g., 12, chap. 3]:

I. The MUltIPLIER RULE. A set of multipliers $l_{0}, l_{\beta}(x), e_{\mu}$ for an admissible $\operatorname{arc} E$ is a set for which the $l_{0}, e_{\mu}$ are constants and the functions $l_{\beta}(x)$, defined on the interval $x_{1} x_{2}$ belonging to $E$, are continuous except possibly at values of $x$ defining corners of $E$ at which they nevertheless have well-defined forward and backward limits. The arc $E$ satisfies the multiplier rule if there exist constants $c_{i}$ and multipliers $l_{0}, l_{\beta}(x), e_{\mu}$ such that for $F=l_{0} f+l_{\beta}(x) \phi_{\beta}$ the equations

$$
F_{y_{i^{\prime}}}=\int_{x_{1}}^{x} F_{y_{i}} d x+c_{i}, \quad \phi_{\beta}=0
$$

are satisfied along $E$, and furthermore such that the end values of $E$ satisfy the equations

$$
\left[\left(F-y_{i}^{\prime} F_{y_{i}^{\prime}}\right) d x+F_{y_{i}^{\prime}} d y_{i}\right]_{1}^{2}+l_{0} d g+e_{\mu} d \psi_{\mu}=0, \quad \psi_{\mu}=0
$$

identically in the differentials $d x_{s}, d y_{i s}$. 
It has been proved [12, p. 27] that the identically vanishing set of multipliers is the only set having constants $l_{0}, e_{\mu}$ all zero, or having functions $l_{0}, l_{\beta}(x)$ which vanish simultaneously at some value $x$ on the interval $x_{1} x_{2}$.

$\mathrm{II}_{N}^{\prime}$. An admissible arc $E$ satisfies the strengthened condition of Weierstrass if for every set of the type $\left(x, y, y^{\prime}, l\right)$ in a neighborhood $N$ of those belonging to $E$ the inequality

$$
E\left(x, y, y^{\prime}, l, Y^{\prime}\right)>0
$$

is satisfied for all admissible sets $\left(x, y, Y^{\prime}\right) \neq\left(x, y, y^{\prime}\right)$, where

$$
E=F\left(x, y, Y^{\prime}, l\right)-F\left(x, y, y^{\prime}, l\right)-\left(Y_{i}^{\prime}-y_{i}^{\prime}\right) F_{y_{i^{\prime}}}\left(x, y, y^{\prime}, l\right) .
$$

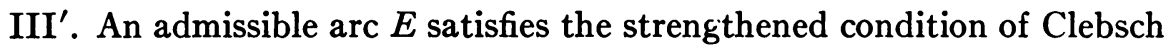
if at every element $\left(x, y, y^{\prime}, l\right)$ belonging to $E$, the inequality

$$
F_{y^{\prime} y^{\prime}}\left(x, y, y^{\prime}, l\right) \pi_{i} \pi_{k}>0
$$

is satisfied for all non-vanishing sets $\pi_{i}$ satisfying the equations

$$
\phi_{\beta y_{i}{ }^{\prime}}\left(x, y, y^{\prime}\right) \pi_{i}=0 .
$$

If we represent by $q, q_{\mu}$ the quadratic forms in $d x_{s}, d y_{i s}$ whose coefficients are the second derivatives of the functions $g, \psi_{\mu}$, respectively, the second variation of $J$ for an extremal arc $E$ with multipliers $l_{0}=1, l_{\beta}(x), e_{\mu}$ has the value

in which

$$
J_{2}(\xi, \eta)=2 \gamma\left[\xi_{1}, \eta\left(x_{1}\right), \xi_{2}, \eta\left(x_{2}\right)\right]+\int_{x_{1}}^{x_{2}} 2 \omega\left(x, \eta, \eta^{\prime}\right) d x
$$

$$
\begin{aligned}
& 2 \omega=F_{y_{i} y_{k} \eta_{i} \eta_{k}}+2 F_{y_{i} \nu_{k}{ }^{\prime} \eta_{i} \eta_{k}{ }^{\prime}+F_{y_{i}{ }^{\prime} \nu_{k}} \eta_{i}^{\prime} \eta_{k}^{\prime}} \\
& 2 \gamma=\left[\left(F_{x}-y_{i}^{\prime} F_{y_{i}}\right) d x+2 F_{y_{i}} d y_{i} d x\right]_{1}^{2}+2 q+2 e_{\mu} q_{\mu}
\end{aligned}
$$

with $d x, d y_{i}$ replaced by $\xi, y_{i}^{\prime} \xi+\dot{\eta}_{i}[12$, p. 71$]$. The equations of variation along $E$ are the equations

$$
\Phi_{\beta}\left(x, \eta, \eta^{\prime}\right)=0, \quad \Psi_{\mu}\left[\xi_{1}, \eta\left(x_{1}\right), \xi_{2}, \eta\left(x_{2}\right)\right]=0
$$

in which

$$
\Phi_{\beta}=\phi_{\beta y_{i}} \eta_{i}+\phi_{\beta y_{i}} \eta_{i}^{\prime},
$$

and $\Psi_{\mu}$ is $d \psi_{\mu}$ with $d x, d y_{i}$ replaced as above by $\xi, y_{i}^{\prime} \xi+\eta_{i}[12$, p. 14]. An admissible set $\xi_{1}, \xi_{2}, \eta_{i}(x)$ is one for which $\xi_{1}, \xi_{2}$ are constants and the functions $\eta_{i}(x)$ have on $x_{1} x_{2}$ the continuity properties of an admissible arc $y_{i}(x)$. The 
second variation $J_{2}(\xi, \eta)$ for $E$ is by definition positive definite if it is positive for all non-vanishing admissible sets $\xi_{1}, \xi_{2}, \eta_{i}(x)$ satisfying the equations (2:3).

$I^{\prime}$. An extremal arc $E$ satisfies the condition $I^{\prime}{ }^{\prime}$ if its second variation is positive definite.

The condition IV' is applicable to an admissible arc which has no corners and satisfies conditions I and III' $^{\prime}$, since such an arc is necessarily non-singular and an extremal [12, pp. 112, 117].

The sufficiency theorem of Hestenes to be considered here is now the following one :

THEOREM 2:1. If an admissible arc $E$ has no corners and satisfies the conditions $\mathrm{I}, \mathrm{II}_{N}^{\prime}, \mathrm{III}^{\prime}, \mathrm{IV}^{\prime}$ with a set of multipliers $l_{0}=1, l_{\beta}(x), e_{\mu}$ then $J(E)$ is a strong relative minimum.

Every admissible arc $E$ satisfies the multiplier rule with none or a limited number of linearly independent non-vanishing sets of multipliers having $l_{0}=0$, It is said to have order of abnormality equal to $q$ if it satisfies I with $q$ and only $q$ such sets $l_{0 \sigma}=0, l_{\beta \sigma}(x), e_{\mu \sigma}(\sigma=1, \cdots, q)$. When $q=0$ it is said to be normal. A set of non-vanishing multipliers with $l_{0}=0$ will be called an abnormal set of multipliers.

For an admissible arc with order of abnormality equal to $q$ the equation

$$
\left[F_{\sigma y_{i}} \eta_{i}\right]_{1}^{2}+e_{\mu \sigma} \Psi_{\mu}=0
$$

with $F_{\sigma}=l_{\beta \sigma}(x) \phi_{\beta}$ is for each $\sigma$ an identity in the variables $\xi_{a}, \eta_{i s}=\eta_{i}\left(x_{s}\right)$, since this is what the first equation $(2: 2)$ becomes for the multipliers $l_{0 \sigma}=0, l_{\beta \sigma}(x)$, $e_{\mu \sigma}$ when the end values of $d x, d y_{i}$ are replaced by those of $\xi, y_{i}^{\prime} \xi+\eta_{i}$. The usual integration by parts applied to the sum

$$
l_{\beta \sigma}(x) \Phi_{\beta}=F_{\sigma y_{i}} \eta_{i}+F_{\sigma y_{i}}{ }^{\prime} \eta_{i}^{\prime}
$$

gives the equation

$$
\int_{x_{1}}^{x_{2}} l_{\beta \sigma} \Phi_{\sigma} d x=\left[F_{\sigma y_{i}} \eta_{i}\right]_{1}^{2}
$$

so that for every admissible set of variations satisfying the equations $\Phi_{\beta}=0$ we find with the help of equations $(2: 4)$ and $(2: 5)$ the relations

$$
\left[F_{\sigma \nu_{i}{ }^{\prime} \eta_{i}}\right]_{1}^{2}=0, \quad e_{\mu \sigma} \Psi_{\mu}=0 .
$$

The matrix of the $q$ sets of values $e_{\mu \sigma}(\sigma=1, \cdots, q)$ is necessarily of rank 
$q$. Otherwise there would be a linear combination of these sets vanishing identically, and, according to a remark made above, the same combination of the linearly independent complete sets $l_{0 \sigma}, l_{\beta \sigma}(x), e_{\mu \sigma}$ would then also vanish identically, which is impossible. In the following paragraphs the variable $\rho$ is understood to have as its range a subset of the numbers $\mu=1, \cdots, p$ such that the determinant $\left|e_{\rho \sigma}\right|$ is different from zero, and the variable $\nu$ will have the range complementary to that of $\rho$. The second equation $(2: 6)$ then shows that for an admissible set $\xi_{1}, \xi_{2}, \eta_{i}(x)$ the equations $\Psi_{\rho}=0$ are consequences of the equations $\Phi_{\beta}=\Psi_{\nu}=0$.

TheOREM 2:2. Let $E$ be an admissible arc without corners which satisfies the hypotheses of Theorem 2:1 with a set of multipliers $l_{0}=1, l_{\beta}(x), e_{\mu}$, and let $\rho$ and $\nu$ be variables whose ranges are determined by the linearly independent abnormal sets of multipliers of $E$ as described in the last paragraph. Then the arc $E$ is normal for the modified problem of minimizing the functional $J(C)+e_{\rho} \psi_{\rho}(C)$ in the class of admissible arcs $C$ satisfying the reduced system of equations $\phi_{\beta}=\psi_{\nu}=0$, and the arc $E$ with the multipliers $l_{0}=1, l_{\beta}(x), e_{\nu}$ satisfies the hypotheses of Theorem 2:1 for the modified problem. Furthermore if $J(E)+e_{\rho} \psi_{\rho}(E)$ is a strong relative minimum for the modified problem, then $J(E)$ is a similar minimum for the original problem.

It is easy to see that the $\operatorname{arc} E$ is normal for the modified problem. For if $E$ had for that problem a set of non-vanishing multipliers of the form $l_{0}=0$, $l_{\beta}(x), e_{\nu}$, the set $l_{0}=0, l_{\beta}(x), e_{\rho}=0, e_{\nu}$ would be multipliers for $E$ and the original problem, necessarily linearly expressible in terms of the $q$ sets $l_{0 \sigma}=0, l_{\beta \sigma}(x)$, $e_{\mu \sigma}(\sigma=1, \cdots, q)$. This is, however, impossible on account of the fact that the determinant $\left|e_{\rho \sigma}\right|$ is not zero.

The arc $E$ satisfies the hypotheses of Theorem 2:1 for the modified problem with the multipliers $l_{0}=1, l_{\beta}(x), e_{\nu}$, as one readily sees by an examination of the conditions $\mathrm{I}, \mathrm{II}_{N}^{\prime}, \mathrm{III}^{\prime}, \mathrm{IV}^{\prime}$. For the condition $\mathrm{IV}^{\prime}$ one needs to note that on account of the second equation $(2: 6)$ the restricted system $\Phi_{\beta}=\Psi_{\nu}=0$ implies the complete system $\Phi_{\beta}=\Psi_{\mu}=0$.

Since the class of arcs in which a minimizing one is sought for the modified problem includes as a subclass those among which a minimizing arc is sought for the original problem, and since on the subclass the values of the functionals $J(C)+e_{\rho} \psi_{\rho}(C)$ and $J(C)$ are equal, the last statement of the theorem is evidently true.

The remarks made at the end of $\$ 1$ are now applicable for the most part to the problem of Bolza also. As a result of Theorem 2:2 it is clear that no generality is lost by proving Theorem 2:1 for normal arcs only, and the proof for such arcs turns out to be in some respects simpler than for the abnormal 
arcs included in the proof of Hestenes. A normal arc is a non-singular arc of the class in which a minimizing arc is sought in the sense that near every normal arc there are an infinity of other arcs of the class [12, pp. 49, 51]. The minimum problem near such an arc is therefore never trivial. Near an abnormal arc $E$, on the other hand, there may be no other arc of the class in which a minimizing one is sought, as in the case when $\psi_{1}(E)$ vanishes and is a strong relative minimum or maximum in the class of admissible arcs satisfying the conditions $\phi_{\beta}=\psi_{2}=\cdots=\psi_{p}=0$. In this case the minimum problem near $E$ is trivial. The variety of types of abnormal arcs is evidently very great. Those included in the sufficiency theorems of Hestenes are of a special type closely related to normal arcs. Other important special types can doubtless be described and discussed, and it might be useful to have results of this kind. But it seems likely that a comprehensive theory would at this time be exceedingly elaborate and difficult, and perhaps impossible.

When the number of the end conditions $\psi_{\mu}=0$ is equal to the number $2 n+2$ of end values $x_{s}, y_{i s}(s=1,2)$ the problem is said to have fixed end points. An admissible arc $E$ is by definition normal on a sub-interval $x^{\prime} x^{\prime \prime}$ if its corresponding sub-arc is normal relative to the problem with fixed end points on that interval. The assumption that an $\operatorname{arc} E$ is normal on every sub-interval is evidently undesirable, for the same reason that it would be undesirable to assume for the problem of $\$ 1$ that the determinants of order $m$ of some particular set belonging to the matrix $\left\|\phi_{\beta y_{i}}\right\|$ are all different from zero. For the problem of Mayer, which is the problem of Bolza with integrand function $f$ identically zero, every minimiżing arc is abnormal on every subinterval, as has been pointed out by Carathéodory [6, 7] and others. No theory based upon the assumption of normality on sub-intervals can therefore be effective in this important case.

\section{BIBLIOGRAPHY}

1. Bolza, Vorlesungen ilber Variationsrechnung, 1st edition, 1909; 2d edition, 1933.

2. Bliss, The problem of Lagrange in the calcnlus of variations, American Journal of Mathematics, vol. 52 (1930), pp. 673-744.

3. Morse, Sufficient conditions in the problem of Lagrange with variable end conditions, American Journal of Mathematics, vol. 53 (1931), pp. 517-546; see also Morse, The problems of Lagrange and Mayer under general end conditions, Proceedings of the National Academy of Sciences, vol. 16 (1930), pp. 229-233.

4. Morse, Sufficient conditions in the problem of Lagrange with fixed end points, Annals of Mathematics, vol. 32 (1931), pp. 567-577.

5. Bliss, The problem of Bolza in the calculus of variations, Annals of Mathematics, vol. 33 (1932), pp. 261-274.

6. Carathéodory, Die Theorie der zweiten Variation beim Problem von Lagrange, Sitzungsberichte der Bayerischen Akademie der Wissenschaften, 1932, pp. 99-114.

7. Carathéodory, Über die Einteilung der Lagrangeschen Variationsprobleme nach Klassen, Commentarii Mathematici Helvetici, vol. 5 (1933), pp. 1-10. 
8. Graves, On the Weierstrass condition for the problem of Bolza in the calculus of variations, Annals of Mathematics, vol. 33 (1932), pp. 747-752.

9. Bliss and Hestenes, Sufficient conditions for a problem of Mayer in the calculus of variations, these Transactions, vol. 35 (1933), pp. 305-326.

10. Hestenes, Sufficient conditions for the general problem of Mayer with variable end points, these Transactions, vol. 35 (1933), pp. 479-490; also Contributions to the Calculus of Variations 1931-1932, The University of Chicago.

11. Hestenes, Sufficient conditions for the problem of Bolza in the calculus of variations, these Transactions, vol. 36 (1934), pp. 793-818.

12. Bliss, The problem of Bolza, Mimeographed lecture notes, University of Chicago, Winter Quarter, 1935.

13. Morse, Sufficient conditions in the problem of Lagrange without assumptions of normalcy, these Transactions, vol. 37 (1935), pp. 147-160.

14. Hestenes, The problem of Bolza in the calculus of variations in parametric form, American Journal of Mathematics, vol. 58 (1936), pp. 391-406.

15. Reid, The theory of the second variation for the non-parametric problem of Bolza, American Journal of Mathematics, vol. 57 (1935), pp. 573-586.

16. Hestenes, On sufficient conditions in the problems of Lagrange and Bolza, Annals of Mathematics, vol. 37 (1936), pp. 543-551.

University of Chicago,

Chicago, Ill. 